\title{
FICOFLÓRULA DO LAGO AMAPÁ EM RIO BRANCO- ACRE, I: EUGLENOPHYCEAE
}

\author{
KEPPELER, E. C., ${ }^{1}$ LOPES, M. R. M. ${ }^{2}$ e LIMA, C. S. ${ }^{3}$ \\ ${ }^{1}$ Mestrado em Ecologia e Manejo de Recursos Naturais/Laboratório de Limnologia, Universidade Federal do Acre - \\ UFAC, Rodovia BR 364 Km 04, CEP 69915-900, Rio Branco, AC, Brasil \\ ${ }^{2}$ Departamento de Ciências da Natureza - UFAC \\ ${ }^{3}$ Laboratório de Limnologia - UFAC \\ Correspondência para: Erlei Cassiano Keppeler, Laboratório de Limnologia, Universidade Federal do Acre, Rua 1B \\ esquina 2A, no 12, Parque das Nações, CEP 69907-270, Rio Branco, AC, Brasil, e-mail: erlei@ hotmail.com \\ Recebido em 19/03/98 - Aceito em 17/11/98 - Distribuído em 22/12/99
}

(Com 36 figuras)

\begin{abstract}
Algal Flora of Lake Amapá in Rio Branco, Acre State, I: Euglenophyceae

The Euglenophyceae flora of Lake Amapá I, Rio Branco, Acre State, Brazil, constitutes a contribution to the phycological inventory of the State of Acre. It is based on the study of 15 samples collected with plankton net and by passing an open flask in areas with dense plankton concentrations. Samples were prepared and preserved with Transeau solution. Twenty five taxons were identified. Genera Euglena and Trachelomonas were the most frequently represented.
\end{abstract}

Key words: plankton, taxonomy of Euglenaceae, Acre State.

\section{RESUMO}

As Euglenophyceae da ficoflórula do Lago Amapá I, Rio Branco, Estado do Acre, Brasil, constituem uma contribuição ao inventário ficológico do Estado do Acre. Está baseada no estudo de 15 amostras coletadas com rede de plâncton e passagem de frasco aberto em meio à massa de algas, as quais foram fixadas e preservadas com solução de Transeau. Foram identificadas 25 Euglenaceae. Os gêneros $E u$ glena e Trachelomonas foram os mais bem representados em nível qualitativo.

Palavras-chave: fitoplâncton, taxonomia de Euglenaceae, Estado do Acre.

\section{INTRODUÇÃO}

O primeiro e único trabalho publicado do estudo de material de algas no Estado do Acre foi o de dois pesquisadores estrangeiros - Archibald \& King (1985). Os autores coletaram em setembro e outubro de 1984, utilizando 28 amostras de dois hábitats distintos do Rio Môa, no município de Cruzeiro do Sul, sendo 11 do Rio Môa propriamente dito e 17 de uma fonte de água sulfurosa, afluente do Rio Môa, registrando a ocorrência de indivíduos pertencentes às divisões Cyanophyta (14), Chlorophyta (37); Bacillariophyta (19) e Euglenophyta (3). Em virtude do pouco conhecimento sobre a composição fitoplanctônica das águas acreanas, especialmente na região de Rio Branco, o presente trabalho visa contribuir com o conhecimento da flora de algas no Estado.

\section{AMBIENTE ESTUDADO}

O Lago Amapá localiza-se no município de Rio Branco, na estrada do Amapá Km 8, Estado do Acre - Brasil, lat. $10^{\circ} 02^{\prime} 36^{\prime \prime} \mathrm{S}$, long. 6750'24'” (Fig. 1).

O lago é um meandro abandonado do Rio Acre, caracterizado por possuir água branca (Sioli, 1968) de aparente ligação com a drenagem principal (Rio Acre). 


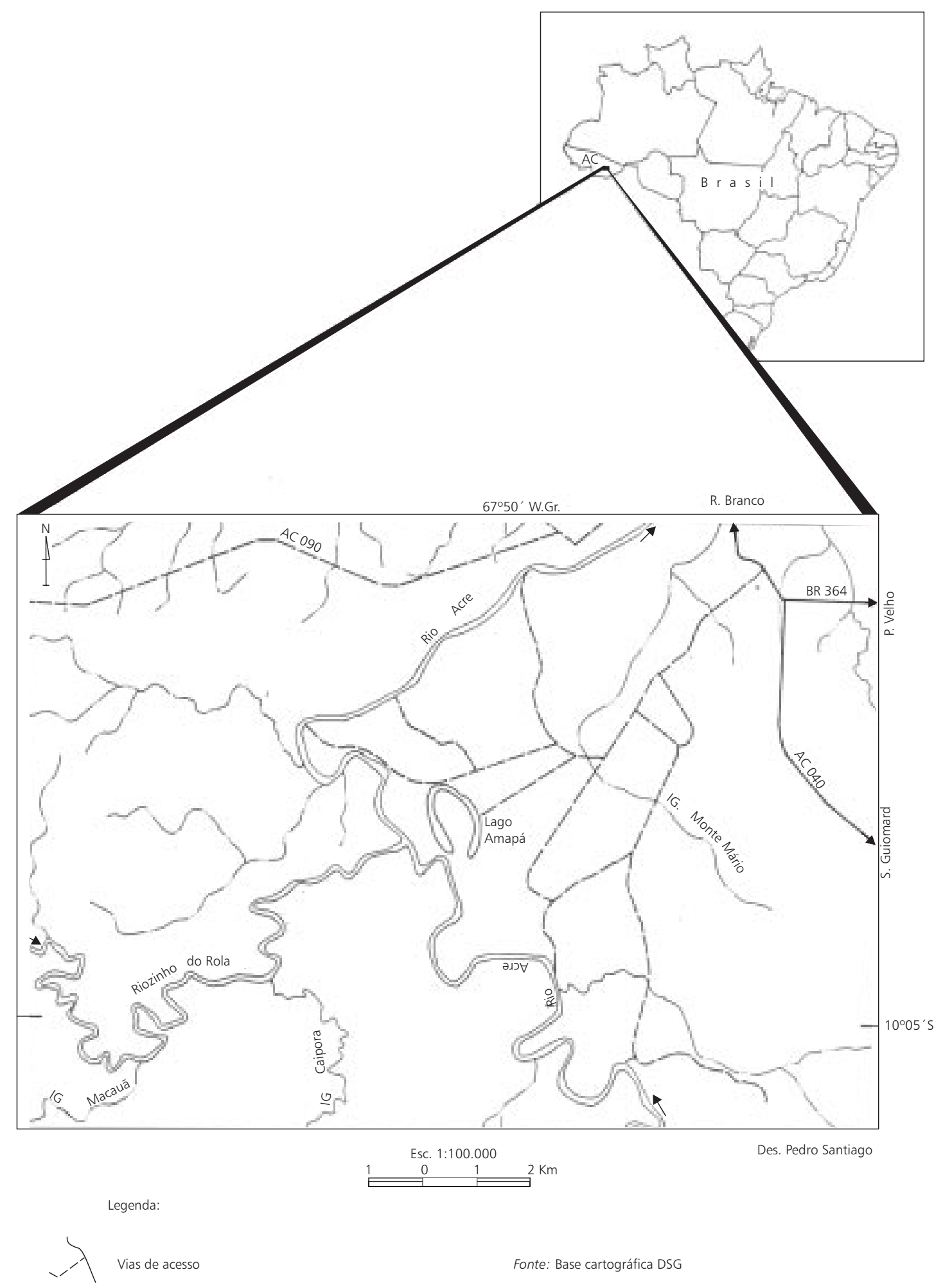

Fig. 1 - Localização do Lago Amapá em Rio Branco, AC, Brasil. 


\section{MATERIAL E MÉTODOS}

A coleta de amostras foi realizada quinzenalmente, no período de março a maio de 1994 , adicionadas de mais três coletas em julho, outubro e novembro de 1994, mensalmente, em uma única estação na região pelágica do lago, perfazendo um total de 15 amostras.

Foram utilizadas duas metodologias de coletas, a saber: (a) concentração do material através de rede de plâncton confeccionada de "nylon", com malha de abertura da ordem de $37 \mu \mathrm{m}$, a qual foi passada várias vezes na camada superficial da água; e (b) simples passagem do frasco aberto em meio à massa de algas eventualmente encontradas na superfície da água e visível a olho nu.

Cada amostra foi, depois de homogeneizada, dividida em duas subamostras, uma das quais foi fixada e preservada com a solução apropriada e a outra, guardada viva em geladeira para observação de características que, eventualmente, seriam afetadas pelo preservativo. As amostras foram armazenadas em frascos de vidro com tampa de polietileno, de pressão, fixadas e preservadas em soluções de Transeau e usada conforme Bicudo \& Bicudo (1970). Tais amostras foram, após o estudo sistemático, depositadas no herbário do Parque Zoobotânico (HPZ) da Universidade Federal do Acre.

$\mathrm{O}$ estudo foi realizado com material vivo e fixado, com o propósito de observar o maior número possível de características de interesse taxonômico. Foi desenvolvido com auxílio de microscópio binocular marca Carl Zeiss Germany composto por câmara-clara e contraste-de-fase acoplados ao sistema óptico do aparelho. A identificação do grupo estudado baseou-se em obras específicas.

\section{RESULTADOS}

\section{Chave para identificação dos táxons encontrados}

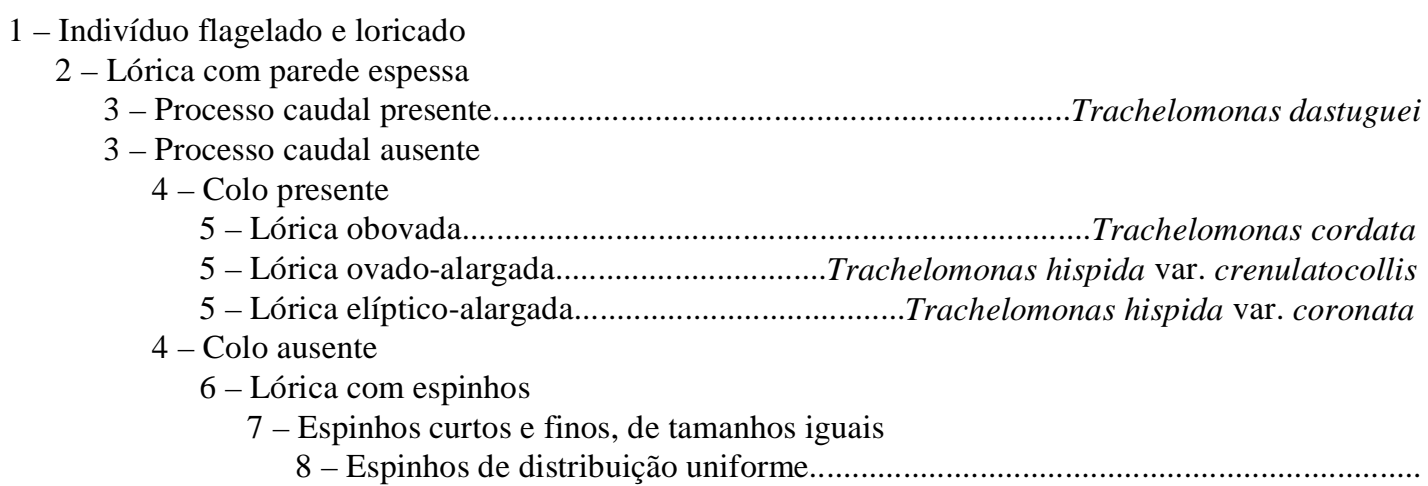
8 - Espinhos de distribuição uniforme.

Trachelomonas superba var. superba

8 - Espinhos concentrados nos pólos.

Trachelomonas superba var. duplex

7 - Espinhos maiores e grosseiros, de tamanhos desiguais 9 - Poucos espinhos apenas em um pólo da lórica

.Trachelomonas armata var. armata f. armata

9 - Numerosos espinhos revestindo ambos os pólos ou toda a lórica.

Trachelomonas armata var. steinii

6 - Lórica sem espinhos

10 - Pólos achatados

11 - Parede celular lisa.

Trachelomonas curta

11 - Parede celular pontuada.......................................Trachelomonas bernardii

10 - Pólos não achatados.................................Trachelomonas volvocina var. punctata

2 - Lórica com parede fina..... Strombomonas maxima

1 - Indivíduo flagelado e não-loricado

12 - Processo caudal presente

13 - Processo caudal longo 
14 - Célula alongada-fusiforme, grãos de paramilo cilíndricos 15 - Célula 98-170 $\mu$ m compr., 2 grãos de paramilo

15 - Célula 240-270 $\mu \mathrm{m}$ compr., 4 grãos de paramilo

Euglena acus var. acus

14 - Célula ovóide, 1 grão de paramilo esférico.

Euglena acus var. longissima Phacus longicauda var. longicauda

13 - Processo caudal curto

16 - Periplasto liso Euglena minuta

16 - Periplasto estriado

17 - Periplasto ornamentado com grânulos brilhantes

17 - Periplasto não-ornamentado com grânulos brilhantes

Euglena spirogyra

18 - Célula levemente ou fortemente metabólica

19 - Levemente metabólica. Euglena oxyuris var. minor 19 - Fortemente metabólica

20 - Célula até $50 \mu \mathrm{m}$ comp. Euglena gracilis

20 - Célula acima de $50 \mu \mathrm{m}$ comp. ........Euglena proxima

18 - Célula não-metabólica

21 - Parede celular com espinhos.... Phacus horridus

21 - Parede celular sem espinhos

22 - Grãos de paramilo em forma de escudos periféricos

.Phacus suecicus

22 - Grãos de paramilo anelares, não periféricos

Phacus curvicauda

12 - Processo caudal ausente

Euglena elastica

23 - Célula metabólica, periplasto liso

23 - Célula não metabólica, periplasto estriado

Lepocinclis texta var. texta

Euglenophyta

Euglenophyceae

Euglenales

Euglenaceae

- Euglena acus Ehrenberg var. acus

Indivíduo solitário, livre-natante, não-metabólico, célula alongada-fusiforme, afilada na parte posterior, terminando em uma cauda fina e longa, 98$170 \times 7-14 \mu \mathrm{m}$, periplasto suavemente estriado longitudinalmente; cloroplastos numerosos, semelhantes a discos; 2 grãos de paramilo longos e cilíndricos (Fig. 2).

- Euglena acus Ehrenberg var. longissima Deflandre

Difere da variedade típica pelo maior comprimento celular (224-270 $\mu \mathrm{m})$ e maior número de grãos de paramilo (quatro) (Fig. 3).

- Euglena elastica Prescott

Indivíduo solitário, livre-natante, fortemente metabólico, alongado, 78-195 × 11-23 $\mu$ m periplasto liso, cloroplastos ovóides e corpos de paramilo numerosos, distribuídos irregularmente na célula (Figs. 4, 5, 6).

- Euglena gracilis Klebs

Indivíduo solitário, livre-natante, fortemente metabólico, fusiforme a ovóide, 37-52 × 12-14,5 $\mu \mathrm{m}$, periplasto estriado, cloroplastos numerosos e discóides (Fig. 8).

- Euglena minuta Prescott

Indivíduo solitário, fortemente metabólico, fusiforme, às vezes piriforme, célula 15-20 × 5-6 $\mu \mathrm{m}$, periplasto liso; cloroplasto semelhante à lâmina com um pirenóide; grãos de paramilo muito pequenos (Figs. 13,14,15).

- Euglena oxyuris Schmarda var. minor Prescott Indivíduo solitário, livre-natante, metabólico, célula alongada-cilíndrica e torcida, 168-180 × 19-25 $\mu \mathrm{m}$, afilando-se abruptamente para a região posterior, processo caudal curto; periplasto estriado; cloroplastos numerosos, semelhantes a discos; grãos de paramilo cilíndricos, um anterior e um posterior (Figs. 9, 10). 


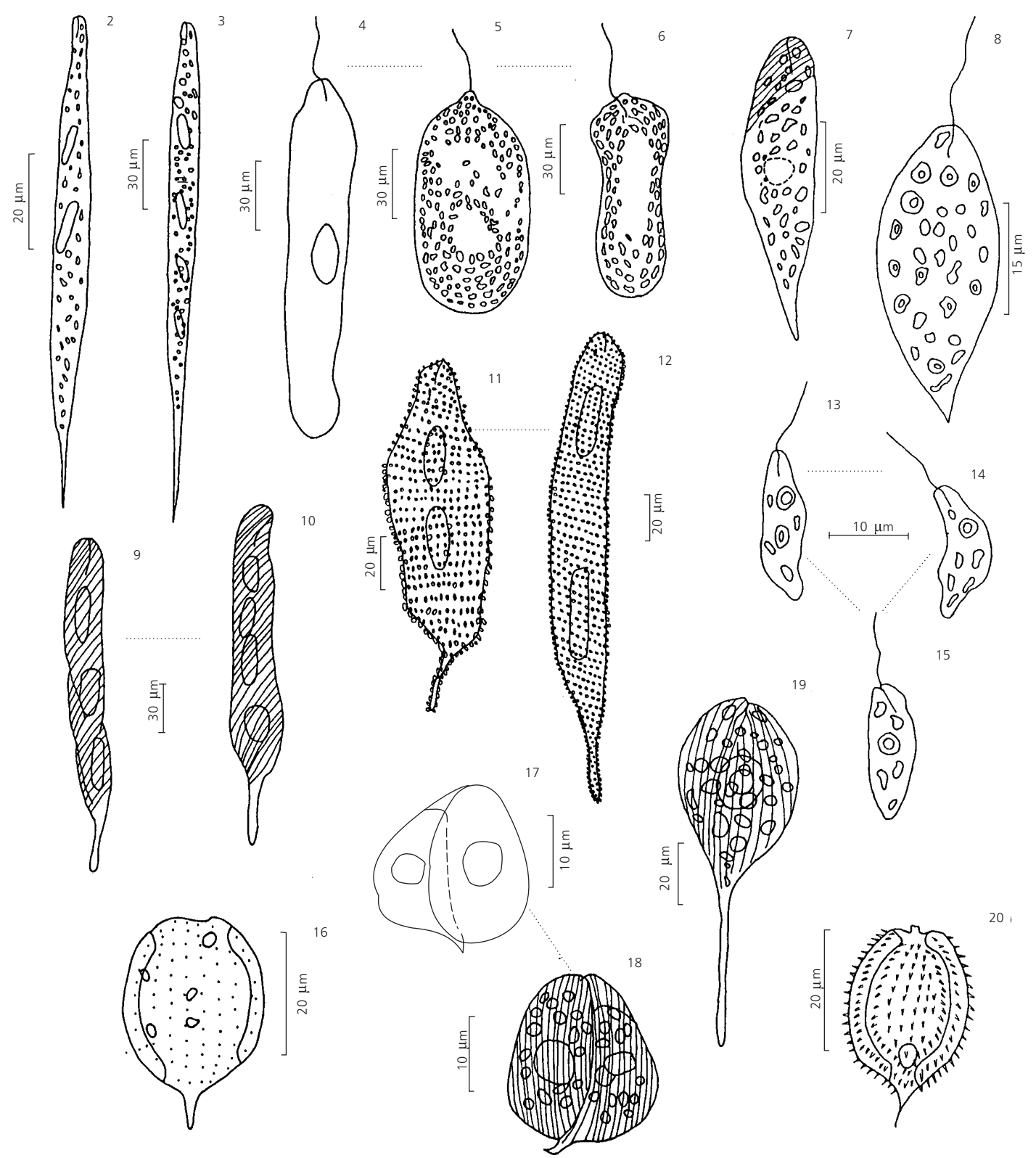

Fig. 2 - Euglena acus Ehrenberg var. acus. Fig. 3 - Euglena acus Ehrenberg var. longissima Deflandre. Figs. 4, 5 e 6 Euglena elastica Prescott. Fig. 7 - Euglena proxima Dangeard. Fig. 8 - Euglena gracilis Klebs. Figs. 9 e 10 - Euglena oxyuris Schmarda var. minor Prescott. Figs. 11 e 12 - Euglena spyrogira Ehrenberg. Figs. 13, 14 e 15 - Euglena minuta Prescott. Fig. 16 - Phacus suecicus Lemmermann. Figs. 17 e 18 - Phacus curvicauda Swirenko. Fig. 19 - Phacus longicauda (Ehrenberg) Düjardin var. longicauda. Fig. 20 - Phacus horridus Pochmann. 


\section{- Euglena proxima Dangeard}

Indivíduo solitário, livre-natante, fortemente metabólico, célula fusiforme, 53-62 × 14-18 $\mu \mathrm{m}$, periplasto estriado, cloroplastos numerosos e discóides, grãos de paramilos dispersos (Fig. 7).

- Euglena spirogyra Ehrenberg

Indivíduo solitário, livre-natante, metabólico, célula alongada-cilíndrica e torcida; afilando-se para a região posterior, $152-208 \times 23-40 \mu \mathrm{m}$, periplasto acastanhado, espiralado, estriado, estrias espiraladas com fileira de pequenos grânulos brilhantes; cloroplastos numerosos, semelhantes a discos; dois grãos de paramilo, um anterior e um posterior (Figs. 11, 12).

- Lepocinclis texta (Düjardin) Lemmermann var. texta

Indivíduo solitário, livre-natante, célula de contorno oval, 45-50 × 36-42 $\mu \mathrm{m}$, pólo posterior amplamente arredondado, processo caudal ausente, periplasto rígido, estriado, cloroplastos numerosos, discóides, parietais, grãos de paramilo numerosos, arredondado a elípticos; estigma volumoso (Fig. 36).

- Phacus curvicauda Swirenko

Indivíduo solitário, livre-natante, célula amplamente ovóide a suborbicular, $31 \times 23 \mu \mathrm{m}$, processo caudal curto e curvado para a esquerda, periplasto liso ou estriado, cloroplastos ovóides (Figs. 17, 18).

- Phacus horridus Pochm.

Indivíduo solitário, livre-natante, $34 \times 20 \mu \mathrm{m}$, células retangulares, em forma de colher, com flancos quase paralelos, pontas um pouco abaixadas; região anterior com elevação em forma de cone, estreitandose para a região posterior, originando um espinho agudo e reto, periplasto com pequenos espinhos enfileirados e direcionados para trás (Fig. 20).

- Phacus longicauda (Ehrenberg) Düjardin var. longicauda

Indivíduo solitário, livre-natante, célula ovóide, 102-120 × 31-39 $\mu \mathrm{m}$, afilada posteriormente no comprimento, processo caudal com a ponta fina, pólo anterior arredondado; periplasto estriado longitudinalmente; flagelo mais curto que a célula em comprimento; cromoplastos numerosos, discóides, parietais, grão de paramilo anelar, central ou posterior; estigma anterior (Fig. 19).

- Phacus suecicus Lemmermann in Pascher \& Lemmermann

Indivíduo solitário, livre-natante, célula amplamente elipsóide ou oval, $36 \times 24,5 \mu \mathrm{m}$, pólo anterior truncado com uma proeminência mediana, processo caudal relativamente longo, ligeiramente desviado ou reto, periplasto estriado longitudinalmente, com fileira de grânulos pontudos, cloroplastos em forma de discos circulares, numerosos, dois corpos de paramilo periféricos e em forma de escudos (Fig. 16).

- Strombomonas maxima (Skvortzov) Deflandre Indivíduo solitário. loricado, lórica fusiforme, 73$85 \times 32-40 \mu \mathrm{m}$, colo presente, cônico, processo caudal terminando em ponta fina, gradualmente, parede celular rugosa, marron-clara, numerosos cromatóforos discóides, grãos de paramilo arrendondados ou alongados (Fig. 35).

- Trachelomonas armata (Ehrenberg) Stein. var. armata fa. armata

Indivíduo solitário, loricado, lórica ovado-alargada, $38-40 \times 32-35 \mu \mathrm{m}$, colo ausente, espessamento anelar presente, parede celular acastanhada, com espinhos cônicos direcionados para baixo, espinhos maiores e fortes localizados no pólo posterior (Fig. 26).

- Trachelomonas armata (Ehrenberg) Stein. var. steinii Lemmermann emend. Deflandre emend. C. Bicudo \& De-Lamonica Freire

Lórica ovado-alargada, $30 \times 23 \mu \mathrm{m}$; difere da variedade típica por apresentar ambos os pólos ou toda a lórica revestida de espinhos, sendo os do pólo posterior sempre mais robustos que os demais (Figs. 27, 28).

- Trachelomonas bernardii Woloszynska

Indivíduo solitário, loricado, lórica oval, $30 \times 40$ $\mu \mathrm{m}$, colo ausente, espessamento anelar presente, pólo apical achatado, parede celular acastanhada, pontuada (Figs. 22, 23, 24)

- Trachelomonas cordata (Drezepolski) Deflandre Indivíduo solitário, loricado, célula cordiforme $27 \times 20 \mu \mathrm{m}$, colo subcilíndrico, curto, $2 \mu \mathrm{m}$ de altura, parede celular lisa (Fig. 25).

- Trachelomonas curta Cunha

Indivíduo solitário, loricado, lórica subesférica, $25 \times 26 \mu \mathrm{m}$, colo ausente; parede celular castanho-amarelada lisa (Fig. 21).

- Trachelomonas dastuguei Balech

Indivíduo solitário, loricado, lórica fusiforme, $95 \times 17 \mu \mathrm{m}$, amplamente inflada na região mediana, um longo e cilíndrico colarinho com 4-6 espinhos fortes na extremidade, processo caudal cônico, com 3-4 espinhos fortes na extremidade, parede celular com espinhos finos e agudos, irregularmente distribuídos (Fig. 29). 

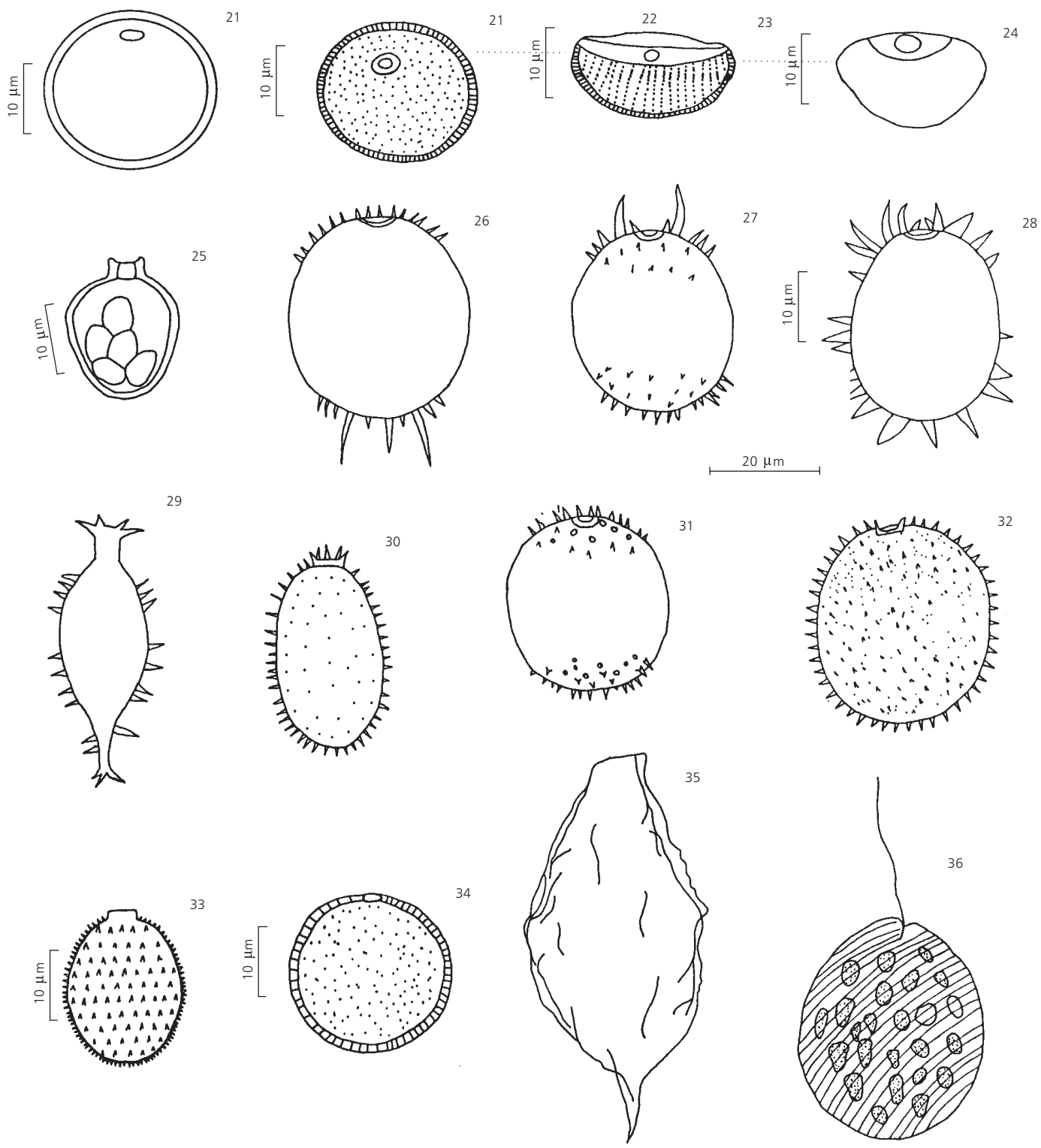

Fig. 21 - Trachelomonas curta Cunha. Figs. 22, 23 e 24 - Trachelomonas bernardii Woloszinska. Fig. 25 - Trachelomonas cordata (Drezepolski) Deflandre. Fig. 26 - Trachelomonas armata (Ehrenberg) Stein var. armata fa. armata. Figs. 27 e 28 - Trachelomonas armata (Ehrenberg) Stein var. steinii Lemmermann emend. Deflandre emend. C. Bicudo \& De Lamonica Freire. Fig. 29 - Trachelomonas dastuguei Balech. Fig. 30 - Trachelomonas hispida (Perty) Stein emend Deflandre var. coronata Lemmermann ex Deflandre. Fig. 31 - Trachelomonas superba Swirenko var. duplex Deflandre. Fig. 32 Trachelomonas superba Swirenko var. superba. Fig. 33 - Trachelomonas hispida (Perty) Stein emend Deflandre var. crenulatocollis (Maskell) Lemmermann. Fig. 34 - Trachelomonas volvocina Ehrenberg var. punctata Playfair. Fig. 35 Strombomonas maxima (Skvortzov) Deflandre. Fig. 36 - Lepocinclis texta (Düjardin) Lemmermann var. texta. 
- Trachelomonas hispida (Perty) Stein emend. Deflandre var. coronata Lemmermann ex. Deflandre

Indivíduo solitário, loricado, lórica oblongo oval, $35-40 \times 19-20 \mu \mathrm{m}$, colo presente, curto, rodeado de espinhos, parede celular com espinhos uniformemente distribuídos (Fig. 30).

- Trachelomonas hispida (Perty) Stein emend. Deflandre var. crenulatocollis (Maskell) Lemmermann

Indivíduo solitário, loricado, lórica ovado-alargada, $24 \times 19 \mu \mathrm{m}$, colo presente, parede celular vermelho-acastanhada, pontuada (Fig. 33).

- Trachelomonas superba Swirenko var. superba Indivíduo solitário, lórica subglobosa, 37-50 × 31-38 $\mu \mathrm{m}$, colo ausente, espessamento anelar presente, parede celular com espinhos curtos e finos, uniformemente distribuídos (Fig. 32).

- Trachelomonas superba Swirenko var. duplex Deflandre

Lórica subglobosa, $30 \times 24 \mu \mathrm{m}$; difere da variedade típica pelos espinhos dos pólos anteriores e posteriores da lórica apresentarem-se maiores que os medianos (Fig. 31).

- Trachelomonas volvocina Ehrenberg var. punctata Playfair

Indivíduo solitário, loricado, lórica globosa, $20 \times$ $19 \mu \mathrm{m}$, colo ausente, espessamento anelar presente, parede celular pontuada, amarelada (Fig. 34 ).

\section{CONCLUSÕES}

O estudo qualitativo no Lago Amapá conduziu-nos às seguintes conclusões: a) Das 15 amostras examinadas, foram determinados deste grupo 25 táxons de Euglenophyceae pertencentes a cinco 5 gêneros, 21 espécies e 7 variedades não típicas.

b) Dos táxons identificados, todos são citados pela primeira vez para o Estado do Acre.

c) O gênero Trachelomonas foi o que apresentou o maior número de táxons (11).

d) Não foi possível a identificação da totalidade dos representantes $(5 \%)$ da comunidade fitoplanctônica devido a amostras populacionais insatisfatórias e a não visualização de vistas taxonômicas fundamentais.

Agradecimentos - Os autores agradecem ao Departamento de Ciências da Natureza - Universidade Federal do Acre, pela utilização das dependências do laboratório e financiamento deste Projeto.

\section{REFERÊNCIAS BIBLIOGRÁFICAS}

ARCHIBALD, P. A. \& KING, J. M., 1985, The algae flora of two distinct habitats along the Moa River in the State of Acre, Brasil. Acta Amazonica, 15(1-2): 145-151.

BICUDO, C. E. M. \& BICUDO, R. M. T., 1970, Algas de águas continentais brasileiras: chave ilustrada para identificação de gêneros. São Paulo, Universidade de São Paulo, 244p.

SIOLI, H., 1968, Principal Biotopes of Primary Production in the Water of Amazonia. In: R. Misra \& B. Gopal (eds.), Proceeding Symp Recent Adv. Tropical Ecology. Varanasi, The International Society for Tropical Ecology, pp. 591600 . 